\title{
BMJ Open Analysis of the sex ratio of reported gonorrhoea incidence in Shenzhen, China
}

\author{
Mingzhou Xiong, ${ }^{1,2}$ Lina Lan, ${ }^{1}$ Tiejian Feng, ${ }^{1}$ Guanglu Zhao, ${ }^{1}$ Feng Wang, ${ }^{1}$ \\ Fuchang Hong, ${ }^{1}$ Xiaobing Wu, ${ }^{1}$ Chunlai Zhang, ${ }^{1}$ Lizhang Wen, ${ }^{1}$ Aizhong Liu, ${ }^{2}$ \\ John McCulloch Best, ${ }^{3}$ Weiming Tang ${ }^{4,5}$
}

To cite: Xiong M, Lan L, Feng T, et al. Analysis of the sex ratio of reported gonorrhoea incidence in Shenzhen, China. BMJ Open 2016;6:e009629.

doi:10.1136/bmjopen-2015009629

- Prepublication history for this paper is available online. To view these files please visit the journal online (http://dx.doi.org/10.1136/ bmjopen-2015-009629).

Received 10 August 2015 Revised 11 November 2015 Accepted 29 December 2015

CrossMark

For numbered affiliations see end of article.

Correspondence to Dr Tiejian Feng; fengtiej@126.com and Lina Lan; 8262268@qq.com

\section{ABSTRACT}

Objective: To assess the clinical process of gonorrhoea diagnosis and report in China, and to determine the difference of sex ratio between reported incidence based on reporting data and true diagnosis rate based on reference tests of gonorrhoea.

Setting: A total of 26 dermatology and sexually transmitted disease (STD) departments, 34 obstetricsgynaecology clinics and 28 urology outpatient clinics selected from 34 hospitals of Shenzhen regarded as our study sites.

Participants: A total of 2754 participants were recruited in this study, and 2534 participants completed the questionnaire survey and provided genital tract secretion specimens. There were 1106 male and 1428 female participants. Eligible participants were patients who presented for outpatient STD care at the selected clinics for the first time in October 2012 were at least 18 years old, and were able to give informed consent.

Outcome measures: Untested rate, true-positive rate, false-negative rate and unreported rate of gonorrhoea, as well as reported gonorrhoea incidence sex ratio and true diagnosis sex ratio were calculated and used to describe the results.

Results: 2534 participants were enrolled in the study. The untested rate of gonorrhoea among females was significantly higher than that among males (female $88.1 \%$, male $68.3 \%, p=0.001$ ). The male-to-female sex ratios of untested rate, true-positive rate, false-negative rate and unreported rate were 1:1.3,1.2:1, 1:1.6 and $1: 1.4$, respectively. The reported gonorrhoea incidence sex ratio of new diagnosed gonorrhoea was 19.8:1 (male vs female: $87 / 1106$ vs $5 / 1420$ ), while the true diagnosis sex ratio was 2.5:1 (male vs female: 161/1106 vs $84 / 1420$ ). These data indicate that the sex ratio of reported gonorrhoea incidence has been overestimated by a factor of $7.9(19.8 / 2.5)$.

Conclusions: We found the current reported gonorrhoea incidence and sex ratios to be inaccurate due to underestimations of gonorrhoea incidence, especially among women.

\section{INTRODUCTION}

Gonorrhoea is a pyogenic infection of the urogenital system caused by Neisseria

\section{Strengths and limitations of this study}

- The reported gonorrhoea incidence is underestimated among both males and females, and this trend is significantly higher among females.

- The reported gonorrhoea incidence sex ratio has been overestimated by a factor of 7.9 .

- Updated diagnostic criteria for gonorrhoea and a set-up active surveillance system in China are suggested to improve the accuracy of reported gonorrhoea incidence.

- False reference results and selection bias may exist, although we have tried our best to avoid them.

gonorrhoeae $(N G)$ that can cause epididymitis and prostatitis in men, and endometritis and pelvic inflammatory disease in women, ${ }^{1}$ and can ultimately lead to infertility in men and women. ${ }^{2}$ Females are more susceptible to $N G$ infection than males due to differences in the structure of the respective reproductive systems, and nearly half of the female infections are asymptomatic. ${ }^{3}{ }^{4}$ According to the WHO estimates in 2008, gonorrhoea is the most frequently sexually transmitted bacterial infection in the world, with approximately 106.1 million new cases diagnosed every year. $^{5}$ In 2012, China reported 95263 new gonorrhoea cases, resulting in an reported incidence of $7.07 / 100000$. The gonorrhoea epidemic was especially prominent in Guangdong, which reported 18014 new cases with a reported incidence of 17.15/ $100,000{ }^{6}$ Over the past several decades, newly reported cases of gonorrhoea within Guangdong province consistently ranked within the top five provinces in China. Shenzhen, a part of the Guangdong province located in south coastal China and adjacent to Hong Kong, has witnessed an alarmingly rapid spread of sexually transmitted diseases (STDs) in recent years. ${ }^{7}$ The reported gonorrhoea incidence in Shenzhen was 51.5/ 
100000 in $2011,{ }^{8}$ which was the highest reported incidence in the entire province.

Interestingly, the reported gonorrhoea incidence among males was much higher than that among females. In 2011, the male-to-female gonorrhoea incidence sex ratio in Shenzhen, Guangdong province and China were 9.9:1, 6.4:1 and 4.3:1, ${ }^{6}{ }^{9}$ respectively.

Gonorrhoea is one of the second-class infectious diseases, according to the Infectious Disease Prevention Act of China. ${ }^{10}$ After the severe acute respiratory syndrome (SARS) epidemic in 2003, the Chinese government built the China Information System for Diseases Control and Prevention (CISDCP) in 2004, and issued "The measures for the management of public health emergencies and infectious disease epidemic surveillance information report" in 2005. ${ }^{11}$ According to this document, gonorrhoea should report to CISDCP mandatorily within $6 \mathrm{~h}$ in urban areas or $12 \mathrm{~h}$ in rural areas. The reported gonorrhoea incidence can be calculated on the basis of reported data. Reported incidence is a very important measure for evaluating the urgency of an epidemic situation, and for establishing prevention and control efforts to combat the disease. However, a number of factors influence the accuracy of reported incidence, including community characteristics and social determinants of health $(\mathrm{SDH}),{ }^{12}{ }^{13}$ as well as potential variability due to biological aspects such as gender.

According to "The measures for the prevention and treatment of sexually transmitted diseases" of China, ${ }^{14}$ each new gonorrhoea case should be reported through CISDCP in hospitals with qualification of STD diagnosis and treatment. The "2007 diagnostic criteria for gonorrhoea in China" indicated, ${ }^{15}$ people who were tested positive for gonorrhoea with or without symptoms could be diagnosed with gonorrhoea. This document recommended Gram stain and isolate culture to test $N G$. Before sampling the urethral swab of a male patient, urination was not allowed at least $2 \mathrm{~h}$. For male patient with symptoms, secrete can be collected using swabs. For an asymptomatic male patient, samples can be collected by placing swabs in urethra for 10-15 seconds. For a female patient, samples can be collected by placing swabs in cervix for 20-30s. Samples can also be obtained by massaging perineal and dip in secrete using swabs. Male patients without complications can be diagnosed conclusively with gonorrhoea with a positive Gram stain microscopy result; the diagnosis of male patients with complications and female patients should be based on the confirmation of $N G$ isolated culture. $N G$ is a very fastidious organism and will perish if not kept in ideal circumstances. The laboratory conditions and the ability of workers would affect the sensitivity and specificity of the test. Nucleic acid amplification tests (NAATs) for gonorrhoea has enabled significant implementation since 2002, creating opportunities for more rapid and accurate gonorrhoea diagnosis. ${ }^{16}$ It has been demonstrated NAATs has a higher sensitivity and specificity than culture and microscopy for gonorrhoea test. ${ }^{17}$ However, this method has recently limited use in some of the big cities in China, due to its high cost and technical requirements. The Guideline for Diagnosis, Treatment and Prevention of Sexually Transmitted Diseases of China, ${ }^{18}$ which was published in 2014 , has regarded this method as one of the recommendatory methods to test $N G$, which has been adopted by the US Food and Drug Administration (FDA).

As the American Centers for Disease Control and Prevention (CDC) estimated, only half of the gonorrhoea cases in America are reported. ${ }^{4}$ Actually, the same problem was also presented or even worse in China. The problem occurred in the process of seeking healthcare in clinics, diagnosis, and case report to CISDCP. First, the physician should believe that the patient has a risk of infection and suggest to the patient to get an $N G$ test done. Second, the patient should accept the physician's suggestion and get the $N G$ test done. Third, the patient got a true positive result. Finally, the physician confirmed that the patient had $N G$ infection, and reported this case to CISDCP. It is possible that the physician did not give the test suggestion, or that the patient was unwilling to get the $N G$ test done, and the false-positive results may also be got by patients. The physician may also have forgotten to report the diagnosed gonorrhoea cases to CISDCP on time. In these instances, the information of these patients with gonorrhoea cannot be obtained from CISDCP. Moreover, some of the individuals may get a false-positive test result and be reported to CISDCP even though they do not have $N G$ infection. The widespread use of CISDCP has underscored the importance of ensuring its accuracy. The discrepancies in symptoms and test methods between males and females may even lead to a different degree of accuracy of male and female reported gonorrhoea incidence.

An inaccurate reported gonorrhoea incidence sex ratio, generated by an inaccurate reported gonorrhoea incidence, may mislead the gonorrhoea prevention policy to target the wrong population for $N G$ screening. To the best of our knowledge, the impact of influence factors in the clinical diagnosis and the reporting process on the reported gonorrhoea incidence sex ratio has not as yet been quantified. It is helpful to find the weakness of disease surveillance system by identifying the influence factors of sex ratio. Thus, we designed and applied a patient survey questionnaire to collect information for gender and sociodemographic characteristics, and collected reporting data of gonorrhoea cases. Furthermore, we performed $N G$ testing on all participants to ascertain gonorrhoea diagnosis. We used rates of untested patients, true-positive rate, false-negative rate and unreported rate to quantify the accuracy of the reported gonorrhoea incidence during diagnosis and the reporting process. Finally, we calculated and compared the reported gonorrhoea incidence sex ratio with the true diagnosis sex ratio, and devised strategies to resolve these discrepancies. 


\section{METHODS}

\section{Patient selection}

Participants were recruited from the dermatology and STD department (STD clinic), obstetrics-gynaecology (OBGYN clinic) and urology outpatient clinic (GUM clinic). A total of 88 clinics, including 26 STD clinics, 34 OBGYN and 28 GUM clinics, were selected from 34 hospitals in Shenzhen. These hospitals were selected of the 144 hospitals that have mandatory reporting of STD cases to CISDCP online within an allotted time and located in the six districts of Shenzhen City. A probability sampling strategy was generated on the basis of patient flow (measured by the average number of STD cases reported to CISDCP in 2009-2011) and administrative level set (municipal level, street level and privately operated). Participants were sampled conveniently and sequentially among those who presented to these clinics for care. Eligible participants were patients who presented for outpatient STD care at the selected clinics for the first time in October, 2012, were at least 18 years old, and were able to give informed consent.

\section{Patient information and sample collection}

Participants were given a questionnaire to collect information on gender and sociodemographic characteristics. Next, a genital tract secretion specimen (urethral swabs from men, vaginal or cervical swabs from women) was collected from each participant and sent to the Shenzhen Center for Chronic Disease Control (SCCDC) to detect $N G$ by nucleic acid testing. The sample collections were performed according to the Guidelines for Laboratory Diagnosis of Sexually Transmitted Diseases of China. ${ }^{19}$ This test result was regarded as the reference test to evaluate the accuracy of $N G$ tests in clinics. After finishing the above procedures, the participant would continue their normal medical service. The clinic physicians then decided on whether or not to collect a second specimen and test for $N G$ in clinics according to their typical diagnostic practices and experiences. Patients with a positive diagnosis of gonorrhoea (according to the second gonorrhoea test result) from the clinics were reported to CISDCP online within 6 or $12 \mathrm{~h}$. The staff at SCCDC compared the original clinical records and reported case information in CISDCP to calculate the unreported rate at the end of the survey.

\section{Laboratory testing}

Clinic laboratories used the methods recommended by 'Diagnostic criteria for gonorrhea' to test the second specimens. Male patients were tested using a Gram stain microscopic smear examination and/or $N G$-isolated culture. Female patients were tested by the $N G$-isolated culture. The clinical laboratory technicians had not been informed that the results of these specimens would be confirmed by SCCDC, in order to exclude the impact of our research work on them. SCCDC used the Roche Cobas Amplicor automated nucleic acid testing system (Roche Diagnostic Systems, Indianapolis, Indiana, USA) to test for the presence of $N G$ of the first collected specimens.

\section{Outcome measurement}

Figure 1 represents the whole process of identifying gonorrhoea cases, from infection to reporting.

Among patients who are infected with NG, a proportion of them would not to seek medical service because of asymptomatic infections or other reasons. These patients would like to choose private clinics or some irregular private hospitals where gonorrhoea cases are not mandatory reported to CISDCP, and the others would go to general hospital which mandatory reporting STD cases to CISDCP. Next, on the basis of the suggestion of physicians and the decision of patients, some of the patients would get the $N G$ test done, and the others would not. Among gonorrhoea patients who were tested for NG, some of them would receive a true-positive result and be diagnosed with gonorrhoea, and the others' results would be false negative. Except them, some of the other people who did not have $N G$ infection but got $N G$ test done and got false-positive results would be wrongly diagnosed with gonorrhoea. Among the diagnosed gonorrhoea cases (including the true positive and false positive), most of them would be reported to CISDCP as required, and some of them would not because of the negligence of physicians. Our study focused on the clinical diagnosis of gonorrhoea and subsequent reporting as presented within the dashed frame.

Stratified analysis was used to analyse patient demographics, which were separated by gender. Untested rate, true-positive rate, false-negative rate and unreported rate of gonorrhoea, as well as reported gonorrhoea incidence sex ratio and true diagnosis sex ratio, were calculated for all participants. The definitions of the various outcomes are listed below:

1. Untested: Participants who were infected with $N G$ and confirmed by reference test, but were not given the $N G$ test in clinics.

2. True positive: The true-positive participants who were diagnosed with gonorrhoea in clinics.

3. False negative: Participants who were infected with $N G$ according to the reference test, but had a negative result in clinics.

4. Unreported: Participants who were diagnosed with gonorrhoea in clinics but not reported to CISDCP on time.

5. Reported gonorrhoea incidence sex ratio: The sex ratio (male vs female) of participants who have been diagnosed with gonorrhoea and reported to CISDCP by physicians.

6. True diagnosis sex ratio: The sex ratio (male:female) of participants with positive $N G$ results on reference test. This excludes the influences of untested rate, true-positive rate, false-negative rate and unreported rate.

\section{Quality control}

Investigators and quality control staff in our study were trained to ensure that their work complied with standards and methods of this survey. Coding labels, 


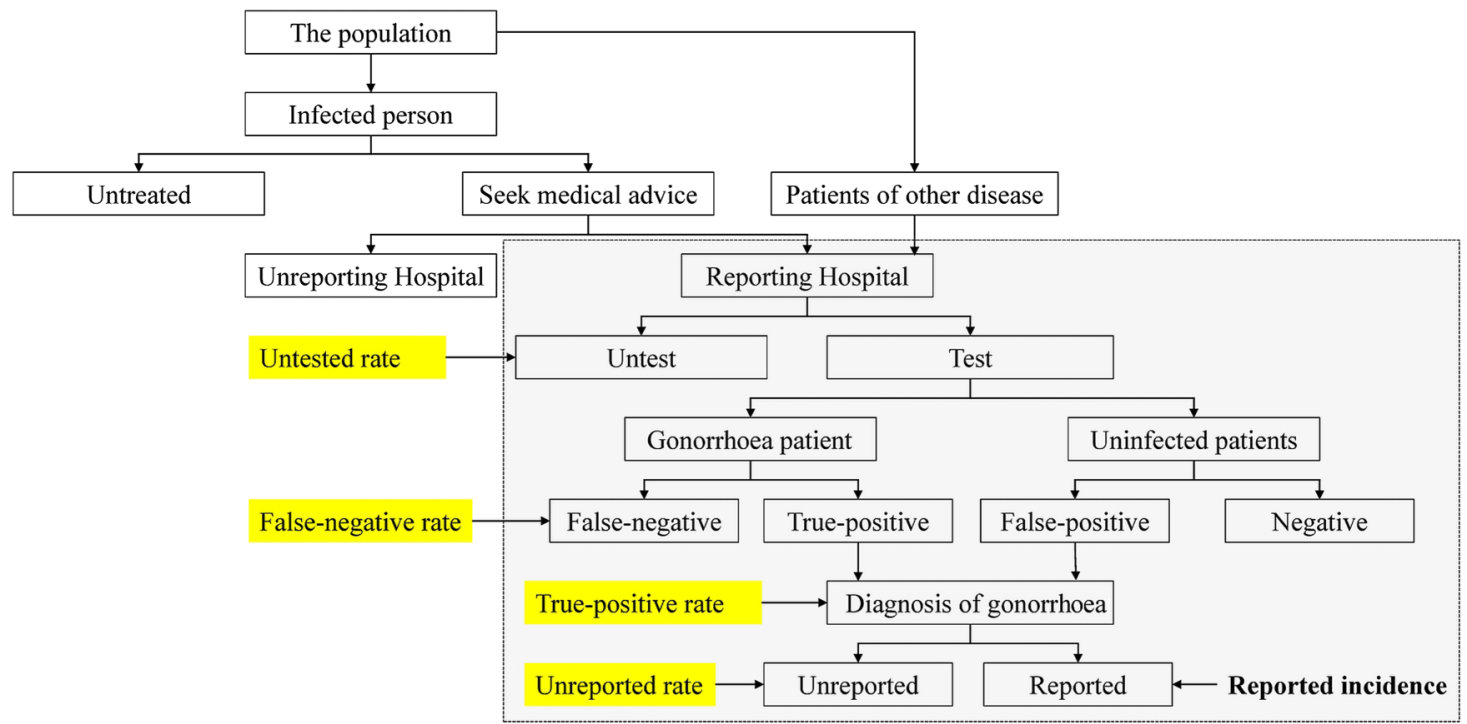

Figure 1 The clinical process of gonorrhoea diagnosis and report in China.

supplies, specimen preservation and transportation strictly abided by the ISO17025 laboratory accreditation standards. The operating procedures were performed in strict accordance with the COBAS AMPLICOR CT/NG Test instructions.

\section{Data analysis}

Epidata 3.0 was used to create the database of patient information. The data were double entered and checked for consistency. Data analysis was performed using SPSS V.17.0. Proportion, rate and ratio were the main statistical indices in our study. The $\chi^{2}$ test and Fisher probabilities in a $2 \times 2$ table data were used to determine statistically significant differences, and the significance level was set at $\mathrm{p}=0.05$.

All participants met with a researcher to discuss the study, and gave written informed consent regarding their inclusion in all parts of the study.

\section{RESULTS}

\section{Patient demographics}

A total of 2754 participants were recruited in this study, and 2534 participants $(92.0 \%)$ completed the questionnaire survey and provided genital tract secretion specimens. There were 1106 male and 1428 female participants. Most of the participants were 20-39 years old $(84.7 \%)$. More female patients were aged 2039 years and had a residence permit outside of Shenzhen $(88.2 \%$ and $80.0 \%$, respectively) than males (80.2\% and $70.6 \%$, respectively). Most of the participants completed a middle school degree $(67.1 \%)$, but more male participants had a bachelor's degree or higher education (32.8\%). Thirty per cent of men $(n=332)$ and $56.2 \%$ of women $(n=803)$ earned less than $¥ 2000 \mathrm{RMB}$ (approximately US\$300) a month. Most of the participants were married (male 68.3\%; female $69.3 \%$ ) (table 1).

\section{Untested rate}

A total of 161 male patients with gonorrhoea (male: $\mathrm{A}+\mathrm{B}+\mathrm{F}$, table 2) were detected by reference test among 1106 male participants (male: $\mathrm{E}+\mathrm{H}$ ). Among them, 51 accepted $N G$ test in clinics (male: $\mathrm{A}+\mathrm{B}$ ), and 110 cases were missed. The resulting untested rate was $68.3 \%$ (95\% CI $61.9 \%$ to $74.7 \%$ ). Among 84 female patients with gonorrhoea (female: $\mathrm{A}+\mathrm{B}+\mathrm{F}$ ) who were diagnosed by reference test among 1420 female participants (female: $\mathrm{E}+\mathrm{H}$ ), 10 accepted the $N G$ test (female: $\mathrm{A}+\mathrm{B}$ ) and 74 cases were missed in clinics. The untested rate among females was $88.1 \%$ (95\% CI $83.2 \%$ to $93.0 \%$ ). The gender difference of untested patients was statistically significant $(p=0.001)$. The sex ratio of untested rate was 1:1.3 (table 2).

\section{True-positive and false-negative rates}

A total of 92 male participants received positive results for the $N G$ test in clinics (male: $\mathrm{A}+\mathrm{C}$ ), and 22 were confirmed by reference test, resulting in a true-positive rate of $23.9 \%$ (95\% CI $17.7 \%$ to $30.0 \%$ ). Among the five female participants who tested positive in clinical settings, only one was confirmed by reference test with a true-positive rate of $20.0 \%$ (95\% CI $0.0 \%$ to $44.8 \%$ ). The gender difference for the true-positive rate was not statistically significant $(p=0.661)$. The male-to-female sex ratio of the true-positive rate was 1.2:1. For false-negative cases, 29 of 51 male participants with positive reference test results had negative results at the clinics $(56.9 \%$, $95 \%$ CI $47.3 \%$ to $66.5 \%)$. Among the $10(\mathrm{~A}+\mathrm{B})$ female patients with gonorrhoea confirmed by reference testing, 9 got false-negative results in clinics $(90.0 \%, 95 \%$ CI $76.9 \%$ to $100.0 \%$ ). The gender difference of the false-negative rate was not statistically significant 
Table 1 The demographic characteristics distribution of the study participants

\begin{tabular}{|c|c|c|c|}
\hline & \multicolumn{2}{|l|}{ Gender } & \multirow{2}{*}{$\begin{array}{l}\text { Total } \\
\text { n (\%) }\end{array}$} \\
\hline & Male n (\%) & Female $\mathrm{n}(\%)$ & \\
\hline \multicolumn{4}{|l|}{ Age (year)* } \\
\hline$\leq 19$ & $12(1.1)$ & 43 (3.0) & $55(2.2)$ \\
\hline 20-39 & $887(80.2)$ & 1259 (88.2) & $2146(84.7)$ \\
\hline$\geq 40$ & $163(14.7)$ & $102(7.1)$ & 265 (10.5) \\
\hline Missing & $44(4.0)$ & $24(1.7)$ & $68(2.6)$ \\
\hline \multicolumn{4}{|l|}{ Census register* } \\
\hline Shenzhen & 308 (27.9) & 270 (18.9) & $578(22.8)$ \\
\hline Outside Shenzhen & $781(70.6)$ & $1142(80.0)$ & 1923 (75.9) \\
\hline Missing & $17(1.5)$ & $16(1.1)$ & $33(1.3)$ \\
\hline \multicolumn{4}{|l|}{ Education* } \\
\hline Primary school and below & $37(3.3)$ & $99(7.0)$ & $136(5.4)$ \\
\hline Middle school & $666(60.2)$ & $1035(72.4)$ & $1701(67.1)$ \\
\hline Bachelor degree or above & $362(32.8)$ & 270 (18.9) & 632 (24.9) \\
\hline Missing & $41(3.7)$ & $24(1.7)$ & $65(2.6)$ \\
\hline \multicolumn{4}{|l|}{ Monthly salary (¥)* } \\
\hline$<2000$ & $332(30.0)$ & 803 (56.2) & $1135(44.8)$ \\
\hline $2000-4000$ & $360(32.5)$ & $363(25.4)$ & 723 (28.5) \\
\hline $4000-6000$ & $206(18.6)$ & $107(7.5)$ & $313(12.4)$ \\
\hline$>6000$ & $172(15.6)$ & $54(3.8)$ & $226(8.9)$ \\
\hline Missing & $36(3.3)$ & $101(7.1)$ & $137(5.4)$ \\
\hline \multicolumn{4}{|l|}{ Marriage } \\
\hline Unmarried & 349 (31.6) & 427 (30.0) & 776 (30.7) \\
\hline Married & 755 (68.3) & $987(69.2)$ & $1742(68.7)$ \\
\hline Divorced or widowed & $2(0.1)$ & $11(0.6)$ & $13(0.5)$ \\
\hline Missing & 0 & $3(0.2)$ & $3(0.1)$ \\
\hline
\end{tabular}

$(p=0.105)$. The false-negative rate sex ratio was $1: 1.6$ (table 2).

\section{Unreported rate}

Among 92 male participants with positive $N G$ results in clinics, five cases were unreported to CISDCP (5.4\%, $95 \%$ CI $2.2 \%$ to $8.6 \%$ ). One case was unreported among five $N G$ positive female participants $(20.0 \%, 95 \%$ CI $0.0 \%$ to $44.8 \%)$. The gender difference of unreported rate was not statistically significant $(\mathrm{p}=0.278)$. The sex ratio of unreported rate was 1:3.7 (table 3 ).

\section{Reported incidence sex ratio versus true diagnosis sex ratio}

Of the 1106 male participants, $7.9 \% \quad(\mathrm{n}=87)$ of cases diagnosed in clinics were reported to CISDCP. Of the 1420 female participants, 4 were reported to the system by physicians, resulting in a reported gonorrhoea incidence of $0.4 \%$. According to the clinic results, the reported gonorrhoea incidence sex ratio was 19.8:1, while the reference test found 161 cases in 1106 male patients and 84 cases in 1420 female patients. However, on the basis of the number of cases detected by the reference laboratory, the true diagnosis rates for male and female participants were $14.6 \%$ and $5.9 \%$, respectively. The true diagnosis sex ratio was 2.5:1. Compared to the true diagnosis sex ratio, the reported incidence sex ratio has been overestimated by a factor of 7.9.

\section{DISCUSSION}

Understanding how the reported incidence of disease is measured and documented is critical to devising strategies for treating diseased populations. Gonorrhoea, while treatable, is a communicable disease that poses a continuing problem in certain locations, which could be attributed, at least in part, to inaccurate diagnosis and reporting. In this paper, we provided new insight into the issue of the gender difference of reported gonorrhoea incidence due to the inaccurate diagnostic and reporting process. We found that females were more likely to be untested, get false-negative results in the clinical process, and have a higher proportion of being unreported to CISDCP. The sex ratio of reported newly diagnosed gonorrhoea in Shenzhen has been overestimated by a factor of 7.9 times as compared with the true diagnosis sex ratio.

The gonorrhoea incidence among males and females is underestimated in China. These inaccurate estimates may impede gonorrhoea treatment and control. First, health policymakers may not be aware of the true prevalence and severity of the $N G$ epidemic leading to an inadequate allocation of resources. Second, the inaccurately reported data may lead physicians and public health doctors to put more efforts into screening male patients versus female patients. Third, untreated $N G$ infection can cause pelvic inflammatory disease, or even infertility and ectopic pregnancy in females. This 
Table 2 The gender difference of untested rate, true-positive rate and false-negative rate

\begin{tabular}{|c|c|c|c|c|c|c|c|c|c|c|c|}
\hline & \multicolumn{5}{|c|}{ Tested (n) (clinic result/reference result) } & \multicolumn{3}{|c|}{ Untested (n) } & \multirow[b]{2}{*}{$\begin{array}{l}\text { Untested rate } \\
(\%, 95 \% \mathrm{Cl})^{\star}\end{array}$} & \multirow[b]{2}{*}{$\begin{array}{l}\text { True-positive } \\
\text { rate }(\%, 95 \% \mathrm{Cl}) \dagger\end{array}$} & \multirow[b]{2}{*}{$\begin{array}{l}\text { False-negative rate } \\
(\%, 95 \% \mathrm{Cl}) \ddagger\end{array}$} \\
\hline & $+/+(A)$ & $-/+(\mathrm{B})$ & $+/-(C)$ & $-1-(\mathrm{D})$ & $\begin{array}{l}\text { Total } \\
\text { (E) }\end{array}$ & $+(F)$ & $-(G)$ & $\begin{array}{l}\text { Total } \\
\text { (H) }\end{array}$ & & & \\
\hline Male & 22 & 29 & 70 & 204 & 325 & 110 & 671 & 781 & $68.3(61.9$ to 74.7$)$ & $23.9(17.7$ to 30.0$)$ & 56.9 (47.3 to 66.5$)$ \\
\hline Female & 1 & 9 & 4 & 120 & 134 & 74 & 1212 & $1286 \S$ & 88.1 (83.2 to 93.0$)$ & $20.0(0.0$ to 44.8$)$ & 90.0 (76.9 to 100.0$)$ \\
\hline Total & 23 & 38 & 74 & 324 & 459 & 184 & 1883 & 2067 & 75.1 (71.2 to 79.0$)$ & 23.7 (17.6 to 29.8$)$ & $62.3(53.5$ to 71.1$)$ \\
\hline Ratio & & & & & & & & & $1: 1.3$ & $1.2: 1$ & $1: 1.6$ \\
\hline
\end{tabular}

contributes a large part to the disease burden across the world, especially in developing countries. ${ }^{1}$ Moreover, these undiagnosed cases could become a hidden reservoir of gonorrhoea infection and lead to a much more serious spread of the disease. In recent years, drug-resistant $N G$ has been increasingly common due to the irregular use and abuse of antibiotics. ${ }^{20}$ 'Superbugs', which are resistant to ceftriaxone, have been found in several countries since being identified in Japan in 2011. Timely and standardised treatment is needed to prevent the transmission of 'superbugs'.

As Liu et $a l^{21}$ found in Hefei city (the capital of Anhui province, China), $82 \%$ of patients with gonorrhoea could be identified by clinic laboratories, which was much higher than that in our research. Maybe the different study site and research design have led to the difference in results. Laboratory testing for $N G$ is the key point to find new gonorrhoea cases, especially for asymptomatic infections. Cases reported to CISDCP should be detected positive for gonorrhoea. NG test should be conducted for asymptomatic patients. The current 'Diagnostic criteria for gonorrhea' of China, which were implemented from 15 October 2007 and recommended microscopy and culture to test $N G$, have a large impact on the appearance of gender difference. Compared to culture, microscopy is quick and the result can be obtained in minutes. On the other hand, culture methods usually require 2-7 days (depending on the hospital) to obtain results. ${ }^{22}$ A large number of female patients are unwilling to take such a long time to get an $N G$ test result. Therefore, when female patients present with mild symptoms or non-specific symptoms and seek medical service in a hospital, physicians may opt for empirical antibiotic treatment instead of an $N G$ test. By then, many female patients lost the opportunity to be detected, which explains why female patients have a higher untested rate in our study. Besides that, Gram stain has a relatively low sensitivity (around $70 \%$ in China), while $N G$ culture has a relative high sensitivity (80-95\% in China).$^{23}$ However, culture methods have a high potential for human error. When collecting the sample, $N G$ specimens are easily contaminated by other bacteria and die after separation from the host, ${ }^{24}$ leading to many false negatives. These differences in laboratory and clinical diagnosis of gonorrhoea are likely to have contributed to the underestimation of male and female cases and an inflated male-to-female ratio. Moreover, the inadequate responsibility of the physician and ability of laboratory personnel also have a negative impact on the accuracy of gender difference of reported gonorrhoea incidence.

Some changes need to be made to get a more accurate reported gonorrhoea incidence. First, the way of disease surveillance needs to be improved. Active surveillance is significantly superior on data accuracy than passive surveillance. CISDCP is a passive surveillance system with all data reported by hospitals. The gonorrhoea cases might be under-reported by physicians both in the diagnostic and reporting process. Some active surveillance methods, such as sentinel surveillance and special survey, can avoid the bias due to the underreporting of new cases. Second, it is of great importance to update the diagnostic criteria for gonorrhoea. Gram stain microscopic smear examination and $N G$ isolated culture methods both have flaws as aforementioned. NAATs has not become the recommendatory method for

Table 3 The gender difference of unreported rate

\begin{tabular}{|c|c|c|c|c|c|}
\hline & Reported & Unreported & Total & $\begin{array}{l}\text { Unreported rate } \\
(\%, 95 \% \mathrm{CI})\end{array}$ & p Value* \\
\hline Male & 87 & 5 & 92 & 5.4 (2.2 to 8.6$)$ & 0.278 \\
\hline Female & 4 & 1 & 5 & 20.0 (0.0 to 44.8$)$ & \\
\hline Total & 91 & 6 & 97 & $6.2(2.8$ to 9.6$)$ & \\
\hline Ratio & & & & $1: 1.37$ & \\
\hline
\end{tabular}


gonorrhoea in the current diagnostic criteria. We suggest that the diagnostic criteria should be updated to recommend NAATs as the standard method for gonorrhoea diagnosis. Furthermore, physicians should perform regularly $N G$ screening tests for potential patients, particularly for female patients who are often asymptomatic.

\section{Study limitations}

The reference test method in our study was imperfect, although this device was one of the most accurate $N G$ test systems in China at the time. Thus, false reference results existed. Second, the patient sampling period was only 1 month, leading to a potential selection bias. However, most studies have found no seasonal variation in $N G$ incidence with a single study showing a slight difference throughout the year. ${ }^{25} 26$

\section{Conclusions}

We found the current reported gonorrhoea incidence and sex ratios to be inaccurate due to underestimations of gonorrhoea incidence, especially among women. Physicians' patient assessments should be monitored by the government and/or hospital to ensure that they are in compliance with diagnostic criteria for gonorrhoea and the diagnostic criteria for gonorrhoea should be updated soon. Physicians should recommend $N G$ tests for any patient with potential gonorrhoea, with particular attention being paid to screening female patients as they are often asymptomatic.

\section{Author affiliations}

${ }^{1}$ STD Prevention and Control Department, Shenzhen Center for Chronic Disease Control, Shenzhen, Guangdong, China

${ }^{2}$ Department of Epidemiology and Health Statistics, Public Health School of Central South University, Changsha, Hunan, China

${ }^{3}$ UCSF, School of Medicine, San Francisco, California, USA

${ }^{4}$ Project-China, University of North Carolina, Guangzhou, Guangdong, China

${ }^{5}$ Department of STI Control, Guangdong Provincial Center for Skin Diseases and STIs control, Guangzhou, Guangdong, China

Acknowledgements The authors would like to thank the staff at the Center of Chronic Disease Control in Shenzhen who made many contributions to this study. They would like to thank the physicians and the participants involved in this study for their wonderful cooperation. They also thank Xia Zou and Willa Dong for assisting with language editing.

Contributors TF, FH and LW have designed this research. XW, LL and CZ have implemented this research and collected research data. GZ and FW completed the reference laboratory test. MX and $\mathrm{AL}$ have analysed the research data and written this manuscript. JMB and WT contribute to the writing and language editing of the manuscript.

Funding This research received no specific grant from any funding agency in the public, commercial or not-for-profit sectors.

Competing interests None declared.

Patient consent Obtained.

Ethics approval The ethics Committee of Shenzhen Center for Chronic Disease Control approved this study.

Provenance and peer review Not commissioned; externally peer reviewed.

Data sharing statement Additional data are available by emailing MX (Lewnxiong@163.com).
Open Access This is an Open Access article distributed in accordance with the Creative Commons Attribution Non Commercial (CC BY-NC 4.0) license, which permits others to distribute, remix, adapt, build upon this work noncommercially, and license their derivative works on different terms, provided the original work is properly cited and the use is non-commercial. See: http:// creativecommons.org/licenses/by-nc/4.0/

\section{REFERENCES}

1. Wang XC, Wang $Q Q$, Zheng HY. Sexual transmitted diseases. Beijing: Science Press, 2010:14-15.

2. Peeling RW, Sparing PF. Sexual transmitted diseases: methods and protocols. NJ: Humana Press, 1999:145-6.

3. Judson FN. Gonorrhea. Med Clin North Am 1990;74:1353-66.

4. Walker CK, Sweet RL. Gonorrhea infection in women: prevalence, effects, screening, and management. Int J Womens Health 2011;3:197-206.

5. Serwin AB, Koper M, Unemo M. Gonorrhoea in 21st centuryinternational and Polish situation. Przegl Epidemiol 2014;68:39-44, 127-131.

6. Yue XL, Jiang N, Gong XD. The analysis report of syphilis and gonorrhea epidemic in China, 2012. http://www.ncstdc.org/ (accessed 8 Nov 2015).

7. Zhang JJ, Zhao GL, Wang F, et al. Molecular epidemiology of genita Chlamydia trachomatis infection in Shenzhen, China. Sex Transm Infect 2012;88:272-7.

8. Feng TJ, Liu XL, Hong FC. The STD epidemic and influence factors analysis of Shenzhen, China. Beijing: People's Medical Publishing House, 2012:20-1.

9. Hong X, Sun XF, Liu FY, et al. Epidemiological analysis of sexually transmitted diseases in Guangdong Province from 2004 to 2012. J Diagn Ther Dermato Venereol 2014;21:65-9.

10. National People's Congress. The Infectious Disease Prevention Act (The 17th Presidential Decree of the People's Republic of China). http://www.gov.cn/gongbao/content/2004/content_62975 (accessed 8 Nov 2015).

11. National Health and Family Planning Commission of the People's Republic of China. The measures for the management of public health emergencies and infectious disease epidemic surveillance information report (The 37th order of the Health Ministry of the People's Republic of China). http://www.nhfpc.gov.cn/mohzcfgs/pgz/ 200901/38689 (accessed 8 Nov 2015).

12. Du P, McNutt LA, O'Campo $\mathrm{P}$, et al. Changes in community socioeconomic status and racial distribution associated with gonorrhea rates: an analysis at the community level. Sex Transm Dis 2009;36:430-8.

13. Moonesinghe R, Fleming $\mathrm{E}$, Truman $\mathrm{BI}$, et al. Linear and non-linear associations of gonorrhea diagnosis rates with social determinants of health. Int J Environ Res Public Health 2012;9:3149-65.

14. National Health and Family Planning commission of the People's Republic of China. The measures for the prevention and treatment of sexually transmitted diseases (The 89th order of Health Ministry of the People's Republic of China). http://www.gov.cn/banshi/ 2005-08/02/content_19262.html (accessed 27 Dec 2014).

15. National Health and Family Planning Commission of the People's Republic of China. Diagnostic criteria for gonorrhea. http://www. nhfpc.gov.cn/zwgkzt/s9491/201212/34114 (accessed 8 Nov 2015).

16. Centers for Disease Control and Prevention (USA). Recommendations for the Laboratory-Based Detection of Chlamydia trachomatis and Neisseria gonorrhea-2014. MMWR Morb Mortal Wkly Rep 2014;63:1-17.

17. Barbee LA, Dombrowski JC, Kerani R, et al. Effect of nucleic acid amplification testing on detection of extragenital gonorrhea and chlamydial infections in men who have sex with men sexually transmitted disease clinic patients. Sex Transm Dis 2014;41:168-72.

18. Wang QQ, Liu QZ, Xu JH. The Guideline for Diagnosis, Treatment and Prevention of Sexually Transmitted Diseases. Shanghai: Shanghai Science and Technology Press, 2014:94-5.

19. Ying YP. The Guidelines for Laboratory Diagnosis of Sexually Transmitted Diseases. Shanghai: Shanghai Science and Technology Press, 2007:19-22.

20. Pease E, Desmond N, Ashby J. The effect on Neisseria gonorrhea screening rates in an integrated clinic following the introduction of dual nucleic acid amplification tests. Int J STD AIDS 2013;24:251.

21. Liu H, Detels R, Yin Y, et al. Do STD clinics correctly diagnose STDs? An assessment of STD management in Hefei, China. Int $J$ STD AIDS 2003;14:665-71.

22. Tanaka $M$, Koga $Y$, Nakayama $H$, et al. Antibiotic-resistant phenotypes and genotypes of Neisseria gonorrhoeae isolates in 
Japan: identification of strain clusters with multidrug-resistant phenotypes. Sex Transm Dis 2011;38:871-5.

23. Yan J. Comparison of five methods for Neisseria gonorrhea detection. Hainan Med J 2012;18:84-5.

24. Hassanzadeh P, Mardaneh J, Motamedifar M. Conventional Agar-Based Culture Method, and Nucleic Acid Amplification Test (NAAT) of the cppB Gene for Detection of Neisseria gonorrhea in
Pregnant Women Endocervical Swab Specimens. Iran Red Crescent Med J 2013;15:207-11.

25. Wang $X Y$, Zheng CX. Epidemiological study on gonorrhea in Xi'an between 2005 and 2010. Chin J Hum Sex 2011;8:16-19.

26. Tan NX, Tan GX, Yang LG, et al. Temporal trends in syphilis and gonorrhea incidences in Guangdong province, China. J Infect Dis 2014;209:426-30. 